J. Watanabe

Nagoya Math. J.

Vol. 50 (1973), 227-232

\title{
A NOTE ON GORENSTEIN RINGS OF EMBEDDING CODIMENSION THREE
}

\author{
JUNZO WATANABE
}

1. Let $A=R / \mathfrak{a}$, where $R$ is a regular local ring of arbitrary dimension and $a$ is an ideal of $R$. If $A$ is a Gorenstein ring and if height $\mathfrak{a}=2$, it is easily proved that $A$ is a complete intersection, i.e., $\mathfrak{a}$ is generated by two elements (Serre [5], Proposition 3). Hence Gorenstein rings which are not complete intersections are of embedding codimension at least three. An example of these rings is found in Bass' paper [1] (p. 29). This is obtained as a quotient of a three dimensional regular local ring by an ideal which is generated by five elements, i.e., generated by a regular sequence plus two more elements. In this paper, suggested by this example, we prove that if $A$ is a Gorenstein ring and if height $\mathfrak{a}=3$, then $\mathfrak{a}$ is minimally generated by an odd number of elements. If $A$ has a greater codimension, presumably there is no such restriction on the minimal number of generators for $a$, as will be conceived from the proof.

In the following the basic results of the two famous papers Bass [2] and Matlis [4] are taken for granted.

2. In this paper we shall consider only Noetherian local rings. If $R$ is a local ring with the maximal ideal $\mathfrak{m}$, we sometimes say that the pair $(R, \mathfrak{m})$ is a local ring. Let $R$ be a ring. If $x, y, \cdots, z$ are elements of $R,(x, y, \cdots, z)$ denotes the ideal they generate. For an $R$-module $M$, hd $M$ denotes the homological dimension of $M$ over $R$. If $R$ is a regular local ring, hd $M<\infty$ for any finite $R$-module $M$ and it holds that hd $M+$ $\operatorname{depth} M=\operatorname{dim} R$.

LEMMA 1. Let $R$ be a regular local ring and let $q$ be a primary ideal belonging to the maximal ideal of $R$. Suppose that $\mathfrak{q}=\bigcap_{i=1}^{n} \mathfrak{q}_{i}$ is an irredundant decomposition of $\mathfrak{q}$ by $n$ irreducible ideals $\mathfrak{q}_{i}$. Let

Received September 25, 1972. 
$0 \rightarrow F_{a} \rightarrow F_{d-1} \rightarrow \cdots \rightarrow F_{1} \rightarrow F_{0} \rightarrow 0$ be a minimal free resolution of $R / \mathfrak{q}$. Then the rank of $F_{d}$ is equal to $n$.

Proof. Since depth $R / \mathfrak{q}=0, \operatorname{dim} R=d$. Therefore we have an isomorphism $\operatorname{Ext}_{R}^{d}(R / \mathfrak{q}, R) \cong \operatorname{Hom}_{R}(R / \mathfrak{q}, E)$, where $E$ denotes the injective envelope of the residue class field. (See [2] Theorem 4.1) Thus the rank of $F_{d}$ is equal to the minimal number of generators for $\operatorname{Hom}_{R}(R / \mathfrak{q}, E)$. On the other hand, the injective envelope of the module $\operatorname{Hom}_{R}\left(\operatorname{Hom}_{R}(R / \mathfrak{q}, E), E\right)$ $\cong R / q$ is an $n$ copies of $E$, and in general these two numbers are identical, because a minimal surjection $F \rightarrow \operatorname{Hom}_{R}(R / \mathfrak{q}, E) \rightarrow 0$ with $F$ free gives an essential injection $0 \rightarrow \operatorname{Hom}_{R}\left(\operatorname{Hom}_{R}(R / \mathfrak{q}, E), E\right) \rightarrow \operatorname{Hom}_{R}(F, E)$. (cf. [4] Theorem 2.3 and Theorem 4.2)

Corollary. Let $R$ be a Gorenstein ring and $\mathfrak{q}$ a perfect ideal of grade d. Let $0 \rightarrow F_{a} \rightarrow F_{d-1} \rightarrow \cdots \rightarrow F_{0} \rightarrow 0$ be as in the Lemma. Then the rank of $F_{d}$ is the "type" of the Cohen-Macaulay ring $R / \mathfrak{q}$.

Proof. Let $x_{1}, x_{2}, \cdots, x_{r}$ be a maximal regular sequence for both $R$ and $R / \mathfrak{q}$. Then it is well known that the complex:

$$
0 \longrightarrow F_{d} \otimes R / \mathfrak{d} \longrightarrow F_{d-1} \otimes R / \mathfrak{x} \longrightarrow \cdots \longrightarrow F_{0} \otimes R / \mathfrak{x} \longrightarrow 0
$$

is a minimal free resolution of $R / \mathfrak{q}+\mathfrak{x}$, over $R / \mathfrak{x}$, where $\mathfrak{x}=\left(x_{1}, \cdots, x_{r}\right)$. (To prove this we only have to show the acyclicity, and this can be done by induction on $r$.) Since the isomorphisms used in the proof of Lemma 1 hold for a Gorenstein ring $R / \mathfrak{c}$, the assertion follows.

Lemma 2. Let $A$ be an Artin Gorenstein local ring and $\mathfrak{a}$ and $\mathfrak{b}$ be two ideals of $A$. If $0: \mathfrak{a}=0: \mathfrak{b}$, then $\mathfrak{a}=\mathfrak{b}$.

Proof. Since for any ideal $\mathfrak{a}$ of $A$, we have $0:[0: \mathfrak{a}]=\mathfrak{a}$, the assertion is clear. (cf. [3] Satz 1.44)

LEMMA 3. Let $(R, \mathfrak{m})$ be a local ring and $\mathfrak{q}$ an $\mathfrak{m}$-primary irreducible ideal, and let $y$ be an element of $R$ which is not in q. Assume that $\mathfrak{q}: y=\mathfrak{q}+\left(f_{1}, f_{2}, \cdots, f_{n}\right)$. Then we have $\bigcap_{i=1}^{n}\left[\mathfrak{q}: f_{i}\right]=\mathfrak{q}:\left(f_{1}, \cdots, f_{n}\right)=$ $\mathfrak{q}+(y)$. Moreover the following two conditions are equivalent to each other:

i) the intersection of ideals $\bigcap_{i=1}^{n}\left[q: f_{i}\right]$ is irredundant.

ii) $\left\{f_{1}, f_{2}, \cdots, f_{n}\right\}$ is (a set of representatives of) a minimal generators for the ideal $\mathfrak{q}+\left(f_{1}, f_{2}, \cdots, f_{n}\right)$ modulo $\mathfrak{q}$. 
Proof. The equality $\bigcap_{i=1}^{n}\left[q: f_{i}\right]=\mathfrak{q}:\left(f_{1}, \cdots, f_{n}\right)$ is easily verified (without the assumption that $\mathfrak{q}$ is irreducible and $\mathfrak{m}$-primary). We prove the second equality. It is obvious that $q+(y) \subset q:\left(f_{1}, \cdots, f_{n}\right)$. Assume $z \in \mathfrak{q}:\left(f_{1}, \cdots, f_{n}\right)$. Then $z f_{i} \in \mathfrak{q}$ for each $i$, which implies that $\mathfrak{q}: z \supset \mathfrak{q}: y$. Therefore $\mathfrak{q}:(y)=\mathfrak{q}:(y, z)$, and considering everything modulo $\mathfrak{q}$, we conclude by Lemma 2 that $\mathfrak{q}+(y)=\mathfrak{q}+(y, z)$, which proves $\mathfrak{q}:\left(f_{1}, \cdots, f_{n}\right)$ $\subset \mathfrak{q}+(y)$. (Recall that $R / \mathfrak{q}$ is an Artin Gorenstein ring if and only if $\mathfrak{q}$ is $m$-primary and irreducible.)

To prove the second assertion assume $\bigcap_{i=1}^{n}\left[\mathfrak{q}: f_{i}\right]=\bigcap_{i=2}^{n}\left[\mathfrak{q}: f_{i}\right]$ for instance. Then $\mathfrak{q}:\left(f_{1}, f_{2}, \cdots, f_{n}\right)=\mathfrak{q}:\left(f_{2}, \cdots, f_{n}\right)$, and again by Lemma 2 , $\mathfrak{q}+\left(f_{1}, \cdots, f_{n}\right)=\mathfrak{q}+\left(f_{2}, \cdots, f_{n}\right)$. This shows that ii) implies i). The other implication is immediate.

LEMMA 4. Let a be an irreducible m-primary ideal of a local ring $(R, \mathfrak{m})$. If $\mathfrak{b}$ is another irreducible ideal which contains $\mathfrak{a}$, then there is an element $y$ such that $\mathfrak{b}=\mathfrak{a}: y$. Conversely for any element $y$ of $R$ which is not in a, a:y is irreducible.

Proof. Let $E$ be the injective envelope of $R / \mathrm{m}$. From the canonical epimorphism $R / \mathfrak{a} \rightarrow R / \mathfrak{b} \rightarrow 0$ we obtain a monomorphism $0 \rightarrow \operatorname{Hom}_{R}(R / \mathfrak{b}, E)$ $\rightarrow \operatorname{Hom}_{R}(R / \mathfrak{a}, E)$. Since $R / \mathfrak{a}$ and $R / \mathfrak{b}$ are both self-injective, $\operatorname{Hom}_{R}(R / \mathfrak{a}, E)$ $\cong R / \mathfrak{a}$ and $\operatorname{Hom}_{R}(R / \mathfrak{b}, E) \cong R / \mathfrak{b}$. Therefore the above monomorphism shows the existence of $y$ satisfying $\mathfrak{b}=\mathfrak{a}: y$. An $\mathfrak{m}$-primary ideal $\mathfrak{q}$ is irreducible if and only if $\operatorname{dim}_{k} \operatorname{Hom}_{R}(k, R / \mathfrak{q})=1$, where $k=R / \mathfrak{m}$. Consequently the irreducibility of $\mathfrak{a}: y$ follows immediately from the fact that we can define a monomorphism $R /[a: y] \rightarrow R / \mathfrak{a}$ by $1 \bmod [a: y] \mapsto y$ $\bmod a$.

THEOREM. Let $(R, \mathfrak{m})$ be a regular local ring and $a$ be an ideal of height three, such that $R / \mathfrak{a}$ is a Gorenstein ring. Then a is minimally generated by an odd number of elements.

Proof. We denote by $\mu(I)$ the number of minimal generators of an ideal $I$ of a local ring. With this notation it is easy to see that if $x \in \mathfrak{m}$ is a regular element on $R / \mathfrak{a}$, then $\mu(\mathfrak{a})=\mu(\mathfrak{a}+(x) /(x))$, where $\mathfrak{a}+(x) /(x)$ is an ideal of $R /(x)$. Note also that, in this case, height $a=$ height $\mathfrak{a}+(x) /(x)$ and $R / \mathfrak{a}+(x)=R /(x) / \mathfrak{a}+(x) /(x)$ is a Gorenstein ring. Thus we may assume depth $R / \mathfrak{a}=0$, because whenever depth $R / \mathfrak{a}>0$, there is a regular element on $R / \mathfrak{a}$ in $\mathfrak{m}-\mathfrak{m}^{2}$. This amounts to assuming that 
$\mathfrak{a}$ is $\mathrm{m}$-primary and dimension $R=3$, since $R / \mathfrak{a}$ is a Cohen-Macaulay ring.

Let $\mu(\mathfrak{a})=N=n+3$. If $n=0$, there is nothing to prove. Let $n>0$, and let $\mathfrak{a}=\left(x_{1}, x_{2}, x_{3}, f_{1}, f_{2}, \cdots, f_{n}\right)$, where we may assume that $\left(x_{1}, x_{2}, x_{3}\right)=\mathfrak{x}$ is already $\mathfrak{m}$-primary. Since both $\mathfrak{a}$ and $\mathfrak{x}$ are irreducible, by Lemma 4 , there is $y$ such that $a=\mathfrak{x}: y$.

This $y$ can be chosen in such a way that $x_{2}, x_{3}, y$ is a regular sequence. For suppose that $\mathfrak{p}_{1}, \mathfrak{p}_{2}, \cdots, \mathfrak{p}_{t}$ are the associated primes of $\left(x_{2}, x_{3}\right)$ and that

$$
\begin{array}{ll}
y \notin \mathfrak{p}_{i} & i=1,2, \cdots, s \\
y \in \mathfrak{p}_{i} & i=s+1, \cdots, t .
\end{array}
$$

Since $x_{2}, x_{3}$ is a regular sequence, height $\mathfrak{p}_{i}=2$ for every $i$. If $s=t$, the sequence $x_{2}, x_{3}, y$ is a regular sequence. Let $0 \leqq s<t$ and $D=\mathfrak{x} \cap \mathfrak{p}_{1} \cap$ $\cdots \cap \mathfrak{p}_{s}$. Then $\mathfrak{p}_{s+1} \cup \cdots \cup \mathfrak{p}_{t} \not \supset D$. For any element $z \in D$ such that $z \notin \mathfrak{p}_{s+1} \cup \cdots \cup \mathfrak{p}_{t}, x_{2}, x_{3}, y+z$ is a regular sequence and obviously $\mathfrak{x}: y+$ $z=$ a. From now on $y$ is assumed to be chosen in this way.

We are interested in the ideal $q$ generated by $x_{1}, x_{2}, x_{3}$ and $y$. We assert first that these four elements are a minimal generating set for $\mathfrak{q}$. For if $x_{1} \in\left(x_{2}, x_{3}, y\right), x_{1}=a_{2} x_{2}+a_{3} x_{3}+b y$ with suitable elements $a_{2}, a_{3}, b$. This $b$ is an element of $\mathfrak{x}: y$, so that $b$ is a linear combination of $x_{1}$ and $f_{i}$. But this contradicts the fact that $x_{i}$ and $f_{i}$ form a minimal basis for $a$. The same is true with $x_{2}$ and $x_{3}$. Since it is clear that $y$ cannot be omitted, the above assertion is proved. On the other hand, by Lemma $3, \mathfrak{q}=\bigcap_{i=1}^{n}\left[\mathfrak{x}: f_{i}\right]$, and therefore, by Lemma 1 and Lemma 4, $\operatorname{dim}_{k} \operatorname{Tor}_{3}^{R}(k, R / \mathfrak{q})=n$, where $k=R / \mathfrak{m}$.

Let $0 \rightarrow F_{3} \rightarrow F_{2} \rightarrow F_{1} \rightarrow F_{0} \rightarrow R / \mathfrak{q} \rightarrow 0$ be a minimal free resolution of $R /$ q. So far we have proved that $\operatorname{rank} F_{1}=4$ and $\operatorname{rank} F_{3}=n$. Since $\operatorname{rank} F_{0}=1, \operatorname{rank} F_{2}=N$. We may assume that the homomorphism $F_{1} \rightarrow F_{0}$ is defined by the column vector $\varphi$ such that ${ }^{t} \varphi=\left[x_{1} x_{2} x_{3} y\right]$ (where ${ }^{t} \varphi$ denotes the transposed matrix of $\varphi$ ); if elements of $F_{1}$ are represented by row vectors, their images by $\varphi$ are obtained by the usual matrix product. Let $M$ be a matrix that defines $F_{2} \rightarrow F_{1}$, and let $I_{i}$ be the ideal generated by those elements that appear in the $i$-th column of $\boldsymbol{M}$ $(i=1,2,3,4)$. It is easy to see that these $I_{i}$ depend only on the vector $\varphi$ and not on the choice of $M$. In fact $I_{1}$ is nothing but $\left(x_{2}, x_{3}, y\right): x_{1}$, for example. Note $I_{4}=\mathfrak{a}$. By Lemma 4 and by the choice of $y, I_{1}$ is irreducible. We are going to prove that $\mu\left(I_{1}\right)=N-2$, which completes 
the proof of the theorem by induction on $\mu$ of irreducible m-primary ideals, because the least $\mu$ is three.

Consider the following $N \times 4$ matrix $M_{1}$ :

$$
M_{1}=\left[\begin{array}{rrrr}
-y & 0 & 0 & x_{1} \\
0 & -y & 0 & x_{2} \\
0 & 0 & -y & x_{3} \\
a_{11} & a_{12} & a_{13} & f_{1} \\
\ldots & \ldots & \ldots & \ldots \\
\ldots \ldots & \ldots & \ldots \\
a_{n 1} & a_{n 2} & a_{n 3} & f_{n}
\end{array}\right]
$$

where $a_{i j}$ are elements satisfying $a_{i 1} x_{1}+a_{i 2} x_{2}+a_{i 3} x_{3}+f_{i} y=0$, their existence being a consequence of the assumption that $f_{i} \in \mathfrak{x}: y$. Since each row of $M_{1}$ is in $\operatorname{Ker} \varphi$, there is an $N \times N$ matrix $\boldsymbol{T}$ such that $\boldsymbol{T} \boldsymbol{M}=\boldsymbol{M}_{1}$. This $\boldsymbol{T}$ can be regarded as an $\boldsymbol{R}$-endomorphism of each $I_{i}$. Then since $T$ must be an $R$-automorphism of $I_{4}, T$ is invertible and it follows that $I_{1}=\left(y, a_{11}, a_{21}, \cdots, a_{n 1}\right)$. We want to show that these elements are precisely a minimal basis for $I_{1}$. Let $v$ denote the first and $v_{j}$ the $(3+j)$-th row of $M_{1}$, where $j=1,2, \cdots, n$. Assume for instance $a_{11} \in\left(y, a_{21}, \cdots, a_{n 1}\right)$. Then there are elements $b$ and $c_{j}$ such that the first component of $\boldsymbol{u}=b \boldsymbol{v}$ $+\sum_{j=2}^{n} c_{j} \boldsymbol{v}_{j}$ is $a_{11}$. Set $\boldsymbol{u}^{\prime}=\boldsymbol{v}_{1}-\boldsymbol{u}$. Then the first component of $\boldsymbol{u}^{\prime}$ is 0 , and the fourth component of $\boldsymbol{u}^{\prime}$ has the form $f_{1}-\alpha$, where $\alpha$ is an element of $\left(x_{1}, f_{2}, \cdots, f_{n}\right)$. Let $d_{2}$ and $d_{3}$ be the 2 nd and the 3rd component of $\boldsymbol{u}^{\prime}$ respectively. Since $\boldsymbol{u}^{\prime} \in \operatorname{Ker} \varphi, d_{2}, d_{3}$ and $f_{1}-\alpha$ give a relation of $x_{2}, x_{3}, y$, i.e., $d_{2} x_{2}+d_{3} x_{3}+\left(f_{1}-\alpha\right) y=0$. Since $x_{2}, x_{3}, y$ is a regular sequence, it follows that $f_{1}-\alpha \in\left(x_{2}, x_{3}\right)$, whence $f_{1} \in\left(x_{1}, x_{2}, x_{3}, f_{2}, \cdots, f_{n}\right)$, which is impossible. That $y$ is not superfluous is similarly proved. Q.E.D.

COROLlaRY. Let $R$ be a Gorenstein ring and a be an ideal of homological dimension two. If $R / \mathfrak{a}$ is a Gorenstein ring, then $\mu(\mathfrak{a})$ is odd.

Proof. By the first part of the proof of Corollary to Lemma 1, we may assume that $R / \mathfrak{a}$ is Artinian.

Let $x, y, \mathfrak{q}$ etc. be as in the proof of the theorem. In order to repeat the same argument as before we only have to show that hd $R / \mathfrak{q}$ is finite. But we have an exact sequence : $0 \rightarrow R / \mathfrak{a} \stackrel{\varphi}{\longrightarrow} R / \mathfrak{x} \rightarrow R / \mathfrak{q} \rightarrow 0$, where $\varphi$ is defined by $\varphi(1 \bmod \mathfrak{a})=y \bmod \mathfrak{x}$. Since hd $R / \mathfrak{x}=$ hd $R / \mathfrak{a}=3$, it follows that hd $R / \mathfrak{q} \leqq 3$. (In fact hd $R / \mathfrak{q}=3$, since $R / \mathfrak{q}$ is Artinian.) Q.E.D. 
Remark. It can be proved that over a local ring $R$ the existence of an ideal $\mathfrak{a}$ of finite homological dimension such that $R / \mathfrak{a}$ is a Gorenstein ring implies that $R$ itself is a Gorenstein ring. Therefore in the above corollary the condition that $R$ is a Gorenstein ring is unnecessary.

\section{BIBLIOGRAPHY}

[1] Bass, H., Injective dimension in Noetherian rings, Trans. A.M.S., 102 (1962).

[2] Bass, H., On the ubiquity of Gorenstein rings, Math. Zeit., 82 (1963).

[ 3 ] Herzog, H. and E. Kunz, Der kanonische Modul eines Cohen-Macaulay-Rings, Lecture notes in Math., 238, Springer (1971).

[ 4 ] Matlis, E., Injective modules over Noetherian rings, Pacific J. of Math., 8 (1958).

[ 5 ] Serre, J.-P., Sur les modules projectifs, Seminaire Dubreil, 1960/61.

Nagoya University 